\title{
Enhanced Multilevel Hybrid Algorithm for Graph Partitioning
}

\author{
Annu Arora \\ PG Student \\ Department Of Computer Science and \\ Engineering \\ Lovely Professional University, Punjab
}

\author{
Karanvir Kaur \\ Assistant Professor \\ Department Of Computer Science and \\ Engineering \\ Lovely Professional University, Punjab
}

\begin{abstract}
Graph partitioning is a traditional problem with many applications and a number of high quality algorithms have been developed. Graph partitioning by multilevel scheme is more popular, which includes three phases coarsening, initial partitioning and uncoarsening. In this paper a road network graph is partitioned into two parts, with the aim of reducing the cut size. In road network graph, the nodes or vertices are road junctions, endpoint, etc. and the edges are the path or road length. Recently multilevel hybrid graph partitioning algorithm was proposed using local search refinement strategy and balanced big method for initial partition. In this paper simulated annealing is used to improve the quality of partition and has given better results.
\end{abstract}

General terms- Algorithm, graph partitioning, NPhard problem, quality, cut cost.

Keywords- coarsening, road network graph, initial partition, refinement, uncoarsening, simulated annealing.

\section{INTRODUCTION}

Algorithm is a combination of sequence of finite steps to solve a particular problem. Every algorithm takes some data as input and gives some value as output. An algorithm should take zero or more input but should produce at least one output. An algorithm should be deterministic, programming independent and should give correct output. Different data structures are used for storing and managing different types of data. Algorithm can be analyzed by its time and space complexity. Algorithm can be used as a tool for solving a well-defined computational problem. There are different types of problem to be solved. A problem is in $\mathrm{P}$ - class if there exist a deterministic algorithm to solve that problem and that problem can be solvable in polynomial time example Binary search, Quick sort etc. A problem is in NP- class if non-deterministic polynomial algorithm is possible for that problem. A problem is said to be in NP-class if it can be proved in polynomial time. A problem which is NP but not $\mathrm{P}$ is NPcomplete problem. If $\mathrm{G}$ is unknown and $\mathrm{H}$ is NP-complete problem and if $\mathrm{H}$ is polynomially reduced to $\mathrm{G}$ then $\mathrm{G}$ is said to be NP-hard problem. It is easier to harder conversion.

Graph partitioning falls under the category of NP-hard problem. In graph partition problem, a graph $\mathrm{G}$ is given in the form of vertices and edges $\mathrm{G}(\mathrm{V}, \mathrm{E})$ that needs to be partitioned into different smaller graphs. A k-way partition is a technique that divides the graph into $\mathrm{k}$ number of components. A partition is better if there is less number of edges cut between the components. There are various areas where graph partitioning is used example in scientific computing, partitioning in various stages of VLSI design circuit and task scheduling in multi-processor systems.

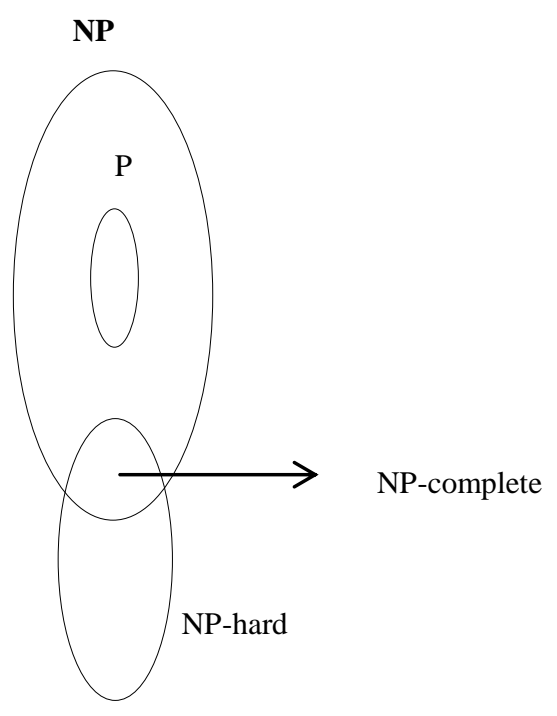

Fig 1.1: NP-complete Problems

Graph partition is important as it is used for making cluster, and detecting cliques in social, pathological and biological networks. There are several graph partitioning techniques exist. For example Breadth first search (BFS) is the simplest graph partitioning technique, which is used to partition the graph into two components $\mathrm{k}=2$.Geometric partitioning method exploits the network flow technique[1] and identify the cut of the graph. Spectral partitioning method is efficient and most widely used technique [2] But due to the fact that it consumes more time in computing Eigen values for the adjacency matrix of the graph it is less in use. A multilevel scheme is a new method for graph partitioning which is based on multilevel paradigm.

A Multilevel graph partitioning algorithm [3] includes three steps. Initially Coarsening the edges and vertices, then partitioning the graph and finally uncoarsen it with refinement to construct the final partition of the graph. Coarsening phase involves transforming the given graph $\mathrm{G}$ into smaller graphs G1, G2, G3...so on these newly formed 
graphs are taken as input for the next phase i.e partitioning phase. Partitioning phase is followed by some refinement strategies to get the finest partition. The last phase is uncoarsening of the graphs it is the reverse of the coarsening phase. After this phase we get the final partition of the original graph. The three phases of multilevel graph partitioning are below:

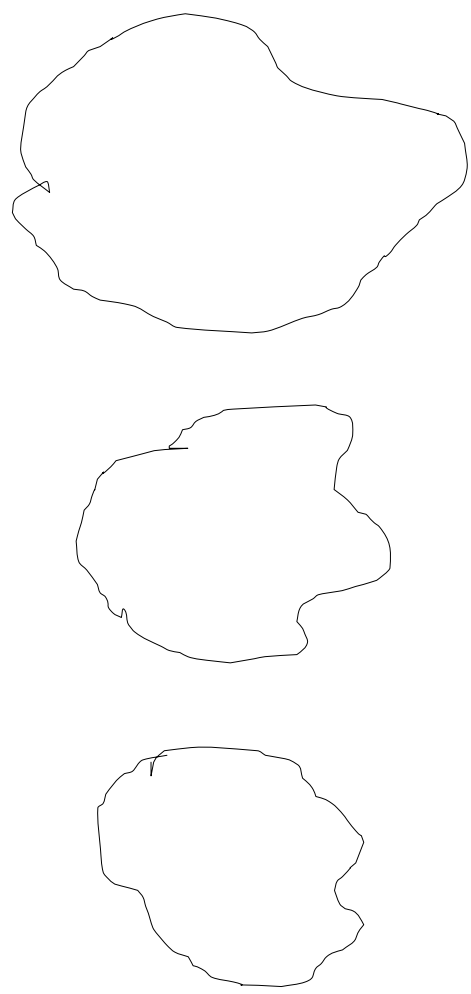

Fig 1.2: Coarsening Phase
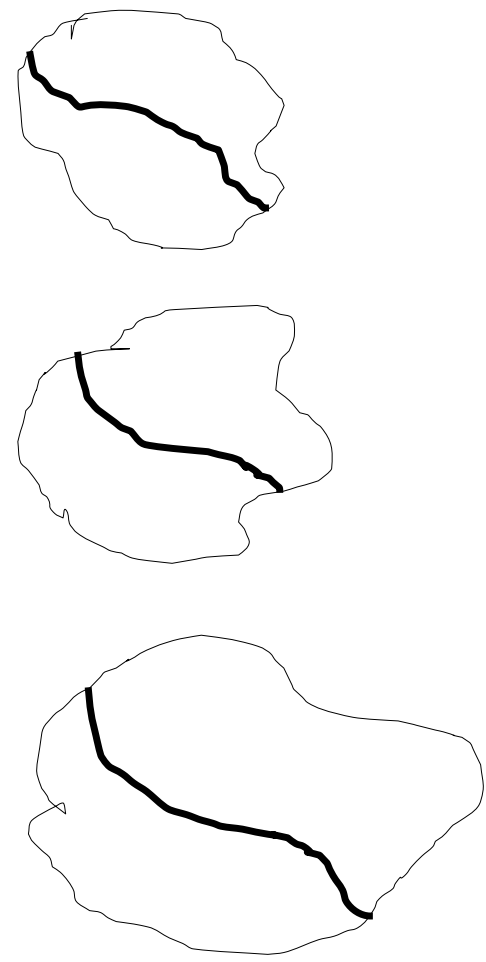

Fig 1.3 Initial Partition and uncoarsening phase

\section{PROBLEM}

A road network graph $\mathrm{G}(\mathrm{V}, \mathrm{E})$ with $\mathrm{V}$ vertices and $\mathrm{E}$ edges is given to be partitioned such that the edge cut cost is minimized. Graph is undirected weighted graph with $\mathrm{w}\left(\mathrm{e}_{\mathrm{ij}}\right)=$ distance between the vertices. Graph partitioning partitions the graph into several partitions $\mathrm{P} 1, \mathrm{P} 2, \mathrm{P} 3 \ldots . . \mathrm{Pk}$ such that size of each partition is balanced.

The edge cut cost $=$ sum of $\mathrm{w}\left(\mathrm{e}_{\mathrm{ij}}\right)$, where $\mathrm{e}_{\mathrm{ij}}$ is the edge between the partition such that one end point is in one partition and another in other partition . $\mathrm{w}\left(\mathrm{e}_{\mathrm{ij}}\right)$ is the weight associated with $\mathrm{e}_{\mathrm{ij}}$

\section{METHODOLOGY}

Enhanced multilevel hybrid graph partitioning algorithm contains four phases.

\subsection{Coarsening phase}

Coarsening phase takes the graph $\mathrm{G}$ as input with $\mathrm{V}$ vertices and $\mathrm{E}$ edges and convert it [3] into smaller graph $\mathrm{G}_{1}, \mathrm{G}_{2}, \mathrm{G}_{3} \ldots .$. so on such that $|\mathrm{V}|>\left|\mathrm{V}_{1}\right|>\left|\mathrm{V}_{2}\right|>\left|\mathrm{V}_{3}\right| \ldots$.... and $|E|>\left|E_{1}\right|>\left|E_{2}\right|>\left|E_{3}\right|$.In this algorithm Heavy Edge Matching [4] is used for coarsening the graph. Matching $\mathrm{M}_{\mathrm{i}}$ is set of edges such that no two edges have same end points on either side. Heavy edge matching considers the heavy edges in the matching set. We use random algorithm to find this matching by visiting the vertices. For any graph $\mathrm{G}(\mathrm{V}, \mathrm{E}), \mathrm{W}\left(\mathrm{e}_{\mathrm{ij}}\right)$ is the sum of the weight of the edges in $\mathrm{G}$

$\mathrm{M}_{\mathrm{i}}=\{$ set of edges $\}$

$w\left(e_{i j}\right)$ is the sum of weight of the edges belonging to $M_{i}$.

The new coarsened graph $G_{i}\left(V_{i}, E_{i}\right)$ have edges $E_{i}=E-M_{i}$ and

$\mathrm{W}_{1}\left(\mathrm{e}_{\mathrm{ij}}\right)=\mathrm{W}\left(\mathrm{e}_{\mathrm{ij}}\right)-\mathrm{w}\left(\mathrm{e}_{\mathrm{ij}}\right)$

The vertices are collapsed in the new coarsened graph using the matching $\mathrm{M}_{\mathrm{i}}$ and the vertices which are not matched are kept as it is in the new graph. Vertices are assigned weight initially and each time when the vertices are collapsed its weight also increases. Vertex weight determines the number of vertices collapsed with it. Heavy Edge Matching is used so that the edges with heavy weight can be avoided from being cut. Coarsening phase is continued until it reaches the coarsening threshold or less than it. Thus in this way coarsening of graph is done to get the final reduced graph.

\subsection{Initial Partition}

Balanced Big method [5] is used for graph partitioning. After the coarsening phase now the coarsened graph needs to be partitioned such that the size of the graph is balanced. The difference between the size of the partition should not be large. Coarsen degree determines the number of vertices in each partition.

According to balanced big method-

I. The vertices with high degree or weight should be assigned to the partition with smaller size.

II. The vertices with low degree or weight are assigned to partition with bigger size. 
We can use BFS, DFS or Random approach for traversing the graph. After partitioning the edge cut cost is computed.

Edge cut cost $=\sum \mathrm{w}\left(\mathrm{e}_{\mathrm{ij}}\right)$,

Such that $\mathrm{e}_{\mathrm{ij}}$ connects the vertex $\mathrm{v}_{\mathrm{i}}$ belonging to partition $\mathrm{P}_{\mathrm{i}}$ and vertex $v_{j}$ belonging to partition $P_{j}$.

\subsection{Refinement}

Refinement is the phase which is used after the initial partition so as to improve the quality of the partition. The main objective of this phase is to reduce the edge cut or the edge cut cost. There are various different techniques for refinement, but in this algorithm Simulated Annealing is used. Simulated Annealing [6] is the optimization approach based on physical annealing, an enhanced version of local optimization. It works under the random number generator and a control parameter called the temperature, cooling ratio $\mathrm{r}$, temperature length $\mathrm{L}$, there are certain generic parameters used as-

INITPROB: it is used to determine the initial temperature for the set of runs. The value of INITPROB is considered as the starting temperature.

TEMPFACTOR: it is also named as cooling ratio $r$ whose value lies between 0 and 1 .

SIZEFACTOR: it is used to set the temperature length $\mathrm{L}$ as $\mathrm{N} *$ SIZEFACTOR, where $\mathrm{N}$ is the size of the expected neighbor.

MINPERCENT: it is used for testing whether annealing run is frozen or not. A counter is maintained which is incremented each time when temperature is reduced and is set to zero when optimum solution is achieved.

\section{Simulated Annealing working:}

1. Find the initial solution $S$.

2. Take an initial temperature $\mathrm{T}>0$.

3. Continue until not frozen

3.1 Perform following steps L times

3.1.1 Find a random neighbor S' of S.

3.1.2 Calculate $\Delta=\operatorname{cost}\left(\mathrm{S}^{\prime}\right)$ - $\operatorname{cost}(\mathrm{S})$

3.1.3 If $\Delta<=0$ (downhill move),

Set $\mathrm{S}=\mathrm{S}^{\prime}$

3.1.4 If $\Delta>0$ (uphill move), set

$\mathrm{S}=\mathrm{S}^{\prime}$ with probability $\mathrm{e}^{-\Delta / \mathrm{T}}$.

3.2 Set $\mathrm{T}=\mathrm{rT}$.

4. Return $\mathrm{S}$.

Here the initial solution $\mathrm{S}$ is taken from the output of the initial partition and the final solution is obtained with improved quality.

\subsection{Uncoarsening Phase}

The final phase is uncoarsening phase, it is reverse of coarsening. The final partition obtained after refinement is projected back to the previously obtained graphs. Here partition is done on G1, G2, G3 ......and finally on the original graph $\mathrm{G}$.

\section{Algorithm: Multilevel Graph Partition (Graph} G)

1. Take the weighted undirected graph as input.

2. While ( $|\mathrm{V}|>$ coarsening threshold) perform Coarsening $\left(\mathrm{G}_{\mathrm{i}}\right.$ to $\left.\mathrm{G}_{\mathrm{i}+1}\right)$

3. For all $\mathrm{v}$ in $\mathrm{V}$ If vertex degree (v) is high perform Balanced partitioning Else Perform Greedy partitioning

4. Simulated annealing(initial partition)

5. While $\left(\mathrm{G}_{\mathrm{i}} !=\mathrm{G}\right)$ perform Uncoarsening $\left(\mathrm{G}_{\mathrm{i}}\right.$ to $\left.\mathrm{G}_{\mathrm{i}-1}\right)$

\section{EXPERIMENTAL RESULTS}

We have taken 15 connected vertices from road network graph of USA from $9^{\text {th }}$ DIMACS implementation challenge. This opened graph is used for partitioning for $\mathrm{k}=2$ and is implemented it in matlab.

For coarsening phase we considered coarsening threshold= 7 , in refining phase for simulated annealing, we set its parameters as INITPROB $=0.2$, TEMPFACTOR $=0.5$, MINPERCENT $=1$ and SIZEFACTOR $=1$. The partition obtained after simulated annealing is compared with hill climbing local search technique and is considered better then it with reduced edge cut cost.

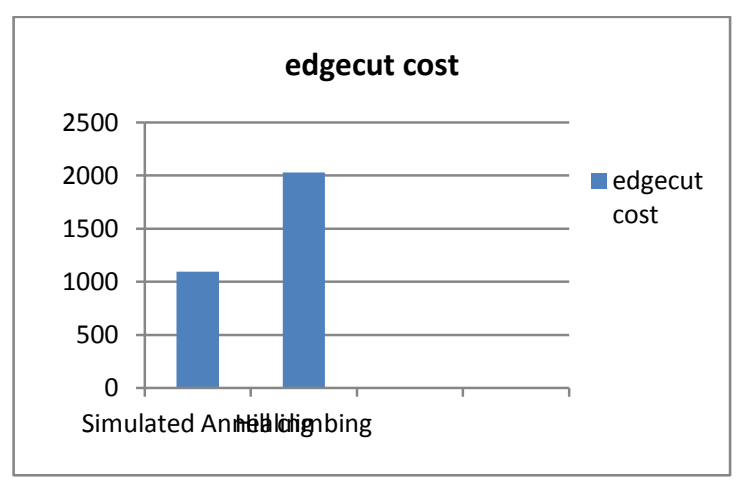

Fig 4.1: Edge cut cost using hill climbing and simulated annealing.

\section{CONCLUSION AND FUTURE SCOPE}

Graph partitioning is basically an NP-hard problem in which a graph $\mathrm{G}(\mathrm{V}, \mathrm{E})$ is to be partitioned into different components such that the cut size is minimized, i.e the number of edges linking to vertices of different components should be minimum. Multilevel graph partitioning is the proper approach for partitioning the graph which includes three stages. In our algorithm we have used simulated annealing which works well for partitioning the graph into two components. The edge cut is better and edge cut cost is reduced than the hill climbing refinement algorithm.

We can use this approach for more number of partitions.Dynamically any graph can be used for partitioning.

\section{ACKNOWLEDGEMENT}

I owe a great many thanks to a great many people who helped and supported me in writing this research paper. 


\section{REFFERENCES}

[1] Sanjeev Arora, Satish Rao, and Umesh Vazirani 2008.Geometry, Flows, and GraphPartitioning Algorithms, communications of the acm October 2008 | vol. $51 \mid$ no. 10.

[2] David S. Johnson 1984.The NP-Completeness Column: An Ongoing Guide Published in J. ALGORITHMS 5, 433-447.

[3] George Karypis and Vipin Kumar 1998. Multilevel kway Partitioning Scheme for Irregular Graphs,Journal Of Parallel And Distributed Computing 48, 96-129 Article No. Pc971404.

[4] George Karypis and Vipin Kumar 1998.A Fast And High Quality Multilevel Scheme For Partitioning Irregular Graphs,Siam J. Sci. Comput. C ${ }^{\circ} 1998$ Society for Industrial And Applied Mathematics Vol. 20, No. 1, Pp. 359-392.

[5] Isabelle Staton, Gabriel Kliot 2012.Streaming Graph Partitioning for Large Distributed Graphs,ACM

[6] David S.Johnson,Cecilia R.Aragon,Lyle A.McGeoch and Catherine schevon 1989. Optimization By Simulated Annealing: An Experimental Evaluation; Part I, Graph Partitioning, Operations Research Society of America ,vol.37,no.6.

[7] Cedric Chevalier and Ilya Safro2009. Comparison of coarsening schemes for multilevel graph partitioning,Sandia National Laboratories, Albuquerque, NM, USA, Ccheval@ Sandia.Gov. Argonne National Laboratory, Argonne, IL, USA, Safro@Mcs.Anl.Gov
[8] Dr.S.Padmavathi, Adline George 2014. Multilevel Hybrid Graph Partitioning Algorithm,department of Computer Science and Engineering Thiagarajar College of Engineering Madurai, India

[9] Dimitris Bertsimas and John Tsitsiklis 1993,SimulatedAnnealing,Statistical sciences vol. 8,no. $1,10-15$

[10] Florian Bourse, Marc Lelarge and Milan Vojnovic 2014, Balanced Graph Edge Partition, Microsoft Reasearch.

[11] L.Tao,Y.C.Zhao,K.Thulasiraman and M.N.S Swamy 1992,Simulated Annealing and Tabu Search Algorithms for Multiway Graph Partition, Journal of circuits,systems and computers

[12] Somayeh Sobati moghadam 2013 ,New algorithm for automatic visualization of metro map, International Journal of Computer Science vol. 10.

[13] Scott Kirkpatrick (1984),Optimization by Simulated Annealing : Quantitative Studies, Journal of Statistical Physics vol 34.

[14] Daniel Delling, Andrew V. Goldberg, Ilya Razenshteyn_x, and Renato F. Werneck 2011. Graph Partitioning with Natural Cuts Microsoft Research Silicon Valley Mountain View, CA, 94043, USA.

[15] Emmanouil Spanakis, Christodoulos Efstathiades, George Pallis Marios and D. Dikaiakos 2011. RealTime Graph Visualization Tool for Vehicular Ad-Hoc Networks, Real-Time Graph Visualization Tool for Vehicular Ad-Hoc Networks . 